This item was submitted to Loughborough's Research Repository by the author.

Items in Figshare are protected by copyright, with all rights reserved, unless otherwise indicated.

Computing the linear complexity for sequences with characteristic polynomial $f^{\wedge} \mathbf{v}$

PLEASE CITE THE PUBLISHED VERSION

http://dx.doi.org/10.1007/s12095-013-0080-3

PUBLISHER

(C) Springer

VERSION

AM (Accepted Manuscript)

LICENCE

CC BY-NC-ND 4.0

REPOSITORY RECORD

Salagean, A.M., Alex J. Burrage, and Raphael C.-W. Phan. 2019. "Computing the Linear Complexity for Sequences with Characteristic Polynomial F^v". figshare. https://hdl.handle.net/2134/12396. 
This item was submitted to Loughborough's Institutional Repository (https://dspace.lboro.ac.uk/) by the author and is made available under the following Creative Commons Licence conditions.

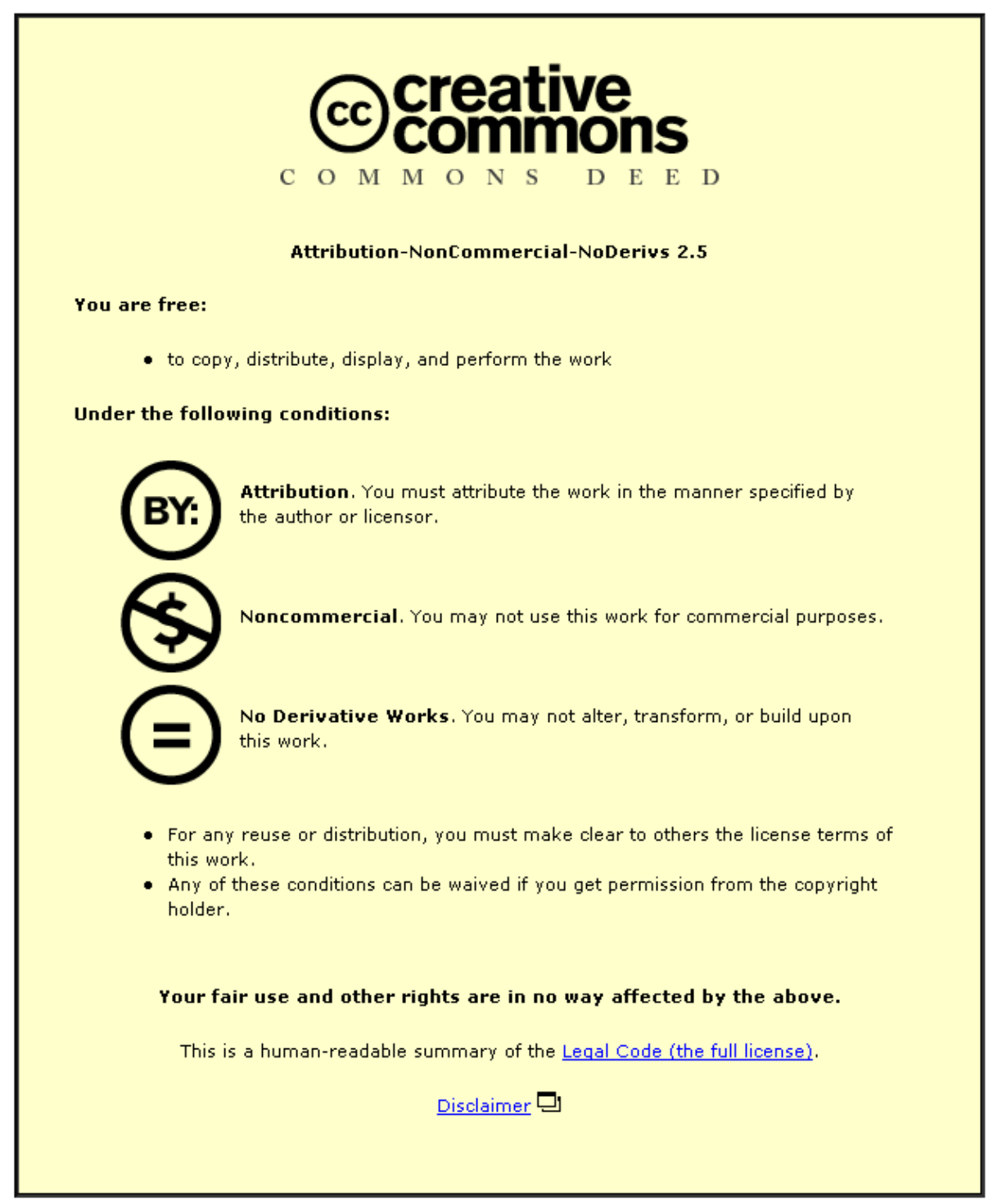

For the full text of this licence, please go to: http://creativecommons.org/licenses/by-nc-nd/2.5/ 


\title{
Computing the Linear Complexity for Sequences with Characteristic Polynomial $f^{v}$
}

\author{
Ana Sălăgean · Alex J. Burrage · Raphael \\ C.-W. Phan
}

the date of receipt and acceptance should be inserted later

\begin{abstract}
We present several generalisations of the Games-Chan algorithm. For a fixed monic irreducible polynomial $f$ we consider the sequences $s$ that have as a characteristic polynomial a power of $f$. We propose an algorithm for computing the linear complexity of $s$ given a full (not necessarily minimal) period of $s$. We give versions of the algorithm for fields of characteristic 2 and for arbitrary finite characteristic $p$, the latter generalising an algorithm of Ding et al. We also propose an algorithm which computes the linear complexity given only a finite portion of $s$ (of length greater than or equal to the linear complexity), generalising an algorithm of Meidl. All our algorithms have linear computational complexity. The proposed algorithms can be further generalised to sequences for which it is known a priori that the irreducible factors of the minimal polynomial belong to a given small set of polynomials.
\end{abstract}

Keywords Linear Complexity · Games-Chan Algorithm · Linear recurrent sequences

\section{Introduction}

The linear complexity of a sequence and the minimum linear recurrence relation (equivalently minimum linear feedback shift register(LFSR)) are important parameters for many applications, including cryptography.

Synchronous stream ciphers typically encrypt by xor-ing the plaintext with a pseudorandom keystream sequence generated from the key and initialisation vector. Known plaintext attacks can recover portions of this keystream sequence, and if the recovered portion consists of a number of successive terms equal to

A preliminary version of this paper was presented at the IEEE International Symposium on Information Theory, St. Petersburg, Russia, August 2011 [1]

Department of Computer Science, Loughborough University, UK, E-mail: A.M.Salagean@lboro.ac.uk · Qualcomm UK, E-mail: aburrage@qti.qualcomm.com · Faculty of Engineering, Multimedia University, Malaysia, E-mail: raphael@mmu.edu.my. This work was done while Alex J. Burrage and Raphael C.-W. Phan were with Loughborough University. 
twice the linear complexity of the sequence, the whole keystream can be recovered efficiently (e.g. using the Berlekamp-Massey algorithm), effectively breaking the cipher. It is therefore essential to ensure that the keystream is a sequence with high linear complexity.

The well-known Berlekamp-Massey algorithm computes the linear complexity of a sequence in quadratic time. For certain classes of sequences more efficient algorithms exist. The Games-Chan algorithm [3] takes linear time and works for binary sequences with period of the form $2^{n}$. It exploits the fact that in this case the minimal polynomial is a factor of $x^{2^{n}}-1=(x-1)^{2^{n}}$ hence it is a power of $x-1$ and one only needs to determine which power. In Algorithm 1 we generalise the Games-Chan algorithm to the case when it is known a priori that the minimal polynomial is a power of a certain fixed irreducible polynomial $f$ (so the GamesChan algorithm would be the case $f=x-1$ ).

The Games-Chan algorithm has been generalised to fields of arbitrary characteristic by Ding et al. in [2] and we will similarly give Algorithm 2 which is the generalisation of Algorithm 1 to arbitrary characteristic. (Our algorithm reduces to the one of Ding et al. in [2] when $f=x-1$.)

The algorithms of Games-Chan and and Ding et al., as well as our first two generalisations mentioned above assume that a whole (not necessarily minimal) period of the sequence is known. By contrast, Berlekamp-Massey algorithm only requires knowledge of a finite portion of consecutive terms of the sequence, of length equal to twice the linear complexity. The latter situation fits in with the cryptanalysis scenario mentioned earlier, where an attacker obtains a finite portion of the sequence and tries to determine the whole sequence. We will give therefore further generalisations of the algorithms of Games-Chan and and Ding et al. which will not require knowledge of the whole period.

It was noted by Sălăgean in [7] and by Meidl in [6] that it is actually not necessary to have a whole period of the sequence in order to determine its linear complexity using the Games-Chan algorithm. It suffices to have a number of terms greater or equal to the linear complexity, provided we still know that the sequence admits as a characteristic polynomial a power of $x-1$ or more generally of some fixed irreducible polynomial $f$. For finite sequences which have a characteristic polynomial of the form $f^{v}$ Meidl gives two algorithms in [6]: one for $f=x-1$ and arbitrary $v$, the other for arbitrary $f$ and $v$ being a power of 2 . We generalise his approach as Algorithm 4, which works for arbitrary $f$ and arbitrary $v$. At first sight it would seem tempting to take this generalisation further, to $k$-error linear complexity, as in [6, Section 4]. However, we do not feel that such work would be worthwhile, as the definition used for the k-error complexity in [6] is a restricted one, (it computes the minimum linear complexity of all sequences $z$ at Hamming distance $k$ from $s$ with the additional condition that $z$ admits as a characteristic polynomial a power of $f$ ) and is not equivalent to the generally used definition (except for $f=x-1$ ). A further explanation is contained in Remark 4.

All the algorithms mentioned so far have linear time complexity, like the Games-Chan algorithm, if we assume the irreducible polynomial is fixed.

We further generalise our algorithms to determine the minimal polynomial when all its irreducible factors are known a priori (Algorithm 5). This algorithm is efficient only if the number of irreducible factors is small and/or their weight is low. 
For all the algorithms and their proofs we found it convenient to use the action of a polynomial on a sequence (Definition 3), a notion that has been used in different guises in several papers. We felt the proofs were shorter and simpler this way compared to the original proofs of many of the algorithms we generalised.

All the Games-Chan type algorithms, including those discussed here, derive their high efficiency from the fact that the minimal polynomial is a (possibly high) power of an irreducible polynomial (or of a small number of irreducible polynomials). We examine now where such sequences could arise in practice. Stream ciphers often use LFSR (Linear Feedback Shift Registers) and NFSR (Non-linear Feedback Shift Registers) as building blocks, and the period of the resulting sequence is derived from the period of these components. Recall that a sequence produced by an LFSR with $n$ registers has period $2^{n}-1$ or a factor thereof, which means its minimal polynomial (being a factor of $x^{2^{n}-1}-1$ ) will only have distinct irreducible factors. On the other hand, a sequence produced by an NFSR has period $2^{n}$ (in which case it is called a de Bruijn sequence) or less, with no obvious divisibility relations between the possible periods (see [4, Section 3.1]). So the minimal polynomial of an NFSR sequence can have multiple factors, in fact the de Bruijn sequences have minimal polynomial $(x-1)^{r}$ for some $r$. Hence, algorithms like the ones in this paper would be relevant to some of the sequences obtained from NFSRs (or a combination of LFSRs and NFSRs) rather than LFSRs. Several of the recent stream cipher proposals (e.g. Grain, Trivium) contain NFSRs.

Algorithms like the ones in this paper, which determine the minimal polynomial when a characteristic polynomial is known, could also be potentially used in cryptography in the following scenario: it may be known from the theoretical analysis that the keystream has a particular characteristic polynomial, but this might not always be minimal, depending on the initial terms, which in turn depend of depending on the key and initialisation vector (IV) used. We would then want to detect the situations where the key/IV produce a sequence with a significantly lower linear complexity than expected.

\section{Preliminaries}

The linear complexity of a sequence is defined as usual:

Definition 1 Given an infinite sequence $s=s_{0}, s_{1}, \ldots$ (or a finite sequence $s=$ $\left.s_{0}, s_{1}, \ldots, s_{m-1}\right)$ with elements in a field $K$ we say that $s$ is a linear recurrent sequence if it satisfies a homogeneous linear recurrence relation, i.e. there are constants $c_{0}, c_{1}, \ldots, c_{L-1} \in K$ such that

$$
s_{j}+c_{L-1} s_{j-1}+\ldots+c_{1} s_{j-L+1}+c_{0} s_{j-L}=0
$$

for all $j=L, L+1, \ldots$ (or for all $j=L, L+1, \ldots m-1$, respectively). We associate to it a characteristic polynomial $g(x)=x^{L}+c_{L-1} x^{L-1}+\ldots+c_{1} x+c_{0}$. If $L$ is minimal for the given sequence, we call $L$ the linear complexity of $s$ and we call $g(x)$ a minimal polynomial.

For infinite sequences the minimal polynomial is unique and any other characteristic polynomial is a multiple of the minimal polynomial.

Throughout this paper we work in a field $K$ of finite characteristic $p$. We denote by $s=s_{0}, s_{1}, \ldots$ an infinite sequence over $K$ and by $s^{\prime}$ a finite sequence consisting 
of successive terms of $s$. The infinite sequence $s$ will be assumed to be periodic with period $N$, i.e. $s_{i}=s_{i+N}$ for all $i \geq 0$. We do not assume $N$ is the minimum period. The finite or infinite sequence consisting only of zeroes will be denoted by $\mathbf{0}$ (the length of the sequence will be clear from the context). A monic irreducible polynomial $f \in K[x]$, different from 1 and $x$ is fixed throughout. For a polynomial $g$ we denote by wt $(g)$ the weight of $g$, i.e. the number of non-zero coefficients of $g$ (by analogy with the Hamming weight of vectors).

For convenience we will introduce the following notation:

Definition 2 Let $g$ be a monic polynomial in $K[x]$. We define $\mathcal{M}(g)$ to be the set of all infinite sequences over $K$ with a characteristic polynomial equal to $g$. We also define $\mathcal{M}\left(g^{\infty}\right)=\cup_{i=0}^{\infty} \mathcal{M}\left(g^{i}\right)$.

The following definition is a commonly used notion:

Definition 3 Let $g=\sum_{i=0}^{n} a_{i} x^{i}$ be a polynomial.

For an infinite sequence $s$ we define the action of $g$ on $s$, denoted $g s$, to be the infinite sequence $t=t_{0}, t_{1}, \ldots$ defined by $t_{i}=\sum_{j=0}^{n} a_{j} s_{i+j}$.

For a finite sequence $s^{\prime}=\left(s_{0}, s_{1}, \ldots, s_{m-1}\right)$ with $m>n$ we define the action of $g$ on $s^{\prime}$, denoted $g s^{\prime}$, to be the finite sequence $t^{\prime}=\left(t_{0}, t_{1}, \ldots, t_{m-n-1}\right)$ defined by $t_{i}=\sum_{j=0}^{n} a_{j} s_{i+j}$. (One could extend the definition to $m \leq n$ but this situation will not occur in this paper).

Using the terminology of actions, the following results concerning characteristic polynomials are immediate:

Lemma 1 Let $g \in K[x]$ be monic and let $s$ be an infinite sequence. Then:

(i) $g$ is a characteristic polynomial of $s$ iff $g s=\mathbf{0}$. Moreover, $g$ is the minimal polynomial of $s$ iff $g$ is a polynomial of minimal degree for which $g s=\mathbf{0}$.

(ii) Let $h \in K[x]$ be monic. If $g$ is the minimal polynomial of $s$ then $g / \operatorname{gcd}(g, h)$ is the minimal polynomial of hs.

(iii) If $s$ is periodic and $N$ is a period of $s$ then $N$ is also a period of $g s$.

Proof Parts (i) and (iii) are clear. For (ii) let $\operatorname{gcd}(g, h)=g_{2}$ and $g=g_{1} g_{2}$. Write $h$ as $g_{2} h^{\prime}$ and denote the minimal polynomial of $h s$ by $g_{3}$. We will prove that $g_{1}=g_{3}$. From (i) we know $g s=g_{1} g_{2} s=\mathbf{0}$ and $g_{3} h s=g_{3} g_{2} h^{\prime} s=\mathbf{0}$. Since the minimal polynomial of a sequence divides any other characteristic polynomial, $g_{1} g_{2} \mid g_{3} g_{2} h^{\prime}$. Since $h^{\prime}$ and $g_{1}$ are coprime we have $g_{1} \mid g_{3}$. On the other hand, $g s=\mathbf{0}$ implies $g h^{\prime} s=g_{1} g_{2} h^{\prime} s=g_{1} h s=\mathbf{0}$ and since $g_{3}$ is the minimal polynomial of $h s$, we have $g_{3} \mid g_{1}$, which combined with $g_{1} \mid g_{3}$ proved previously results in $g_{3}=g_{1}$.

Based on the well-known exponentiation rule (sometimes known as "Freshman's dream"): $(a+b)^{p}=a^{p}+b^{p}$ for all $a, b \in K$, we have:

Lemma 2 Let $g$ be a polynomial over a field $K$ of finite characteristic $p$. Then $\operatorname{wt}\left(g^{p^{w}}\right)=$ $\operatorname{wt}(g)$ for any $w \geq 0$.

Recall that the order of a polynomial $g$ (with $x \nmid g$ ), denoted ord $(g)$ is the smallest integer $m$ such that $g$ is a factor of $x^{m}-1$. The minimal period of a periodic sequence is the same as the order of its minimal polynomial. The order of an irreducible polynomial $f$ is $p^{\operatorname{deg}(f)}-1$ or a factor thereof; hence ord $(f)$ is not divisible by $p$. The order of a power of an irreducible polynomial can be derived as follows: 
Theorem 1 ([5, Theorem 3.8]) Let $f$ be irreducible over $K[x]$ and let $r$ be a positive integer. Then $\operatorname{ord}\left(f^{r}\right)=p^{t} \operatorname{ord}(f)$ where $t$ is the smallest integer with $p^{t} \geq r$.

\section{Linear Complexity of Infinite Sequences which have a Characteristic Polynomial equal to a Power of an Irreducible Polynomial}

We assume that we are given an infinite sequence $s$ of (not necessarily minimal) period $N$ and that we know that the sequence admits as a characteristic polynomial a power of a fixed monic irreducible polynomial $f$, i.e. $s \in \mathcal{M}\left(f^{\infty}\right)$. Our goal is to determine the minimal polynomial of $s$, which will obviously be of the form $f^{r}$ for some integer $r$. A naive algorithm could compute $f^{i} s$ for increasing values of $i$ (by repeatedly replacing $s$ by $f s$ ) until the zero sequence is obtained. Such an algorithm would have complexity $\mathcal{O}(r N)$ if $f$ is considered fixed, or $\mathcal{O}(\operatorname{wt}(f) r N)$ if $f$ is an input parameter. The more efficient method described here finds an upper bound for $r$ and then does a $p$-ary search for the value of $r$. (We will see that this is indeed more efficient, see Theorem 3.)

An upper bound on the value of $r$ can be obtained by looking at the period $N$ and using Theorem 1:

Lemma 3 Let $s \in \mathcal{M}\left(f^{\infty}\right)$ and let $N$ be a (not necessarily minimal) period of s. Write $N$ as $N=p^{w} N^{\prime}$ with $p \nmid N^{\prime}$. Then $s \in \mathcal{M}\left(f^{p^{w}}\right)$, ord $(f) \mid N^{\prime}$ and $p^{w} \operatorname{ord}(f)$ is also a period of $s$.

Proof Let $f^{r}$ be the minimal polynomial of $s$ and let $w^{\prime}$ be the smallest integer with $p^{w^{\prime}} \geq r$. The minimal period of $s$ is $\operatorname{ord}\left(f^{r}\right)$, which by Theorem 1 equals $p^{w^{\prime}} \operatorname{ord}(f)$. Any other period of $s$, for example $N=p^{w} N^{\prime}$ is a multiple thereof. Since neither ord $(f)$ nor $N^{\prime}$ are divisible by $p$, this means $w^{\prime} \leq w$ and $\operatorname{ord}(f) \mid N^{\prime}$. Hence $r \leq p^{w^{\prime}} \leq p^{w}$ so $s \in \mathcal{M}\left(f^{p^{w}}\right)$ and $p^{w} \operatorname{ord}(f)$ is a period of $s$.

Once we have an upper bound for $r$ we can find the exact value of $r$ by a $p$-ary search. We actually find the largest exponent $i$ for which $f^{i} s \neq \mathbf{0}$, i.e. $r-1$. To obtain $r$, a correction of +1 is added at the end. One can view the $p$-ary search equivalently as determining the digits of the base $p$ representation of $r-1$. When testing whether $f^{i} s \neq \mathbf{0}$ for different values of $i$, the values of $i$ which are powers of $p$ are preferred for efficiency reasons, see Lemma 2 .

Lemma 4 Let $s \in \mathcal{M}\left(f^{\infty}\right), s \neq \mathbf{0}$ and let $N=p^{w} N^{\prime}$ with $p \nmid N^{\prime}$ be a (not necessarily minimal) period of $s$ (which by Lemma 3 means $s \in \mathcal{M}\left(f^{p^{w}}\right)$ ). Let $f^{r}$ be the minimal polynomial of $s$. If $w=0$ then $r=1$. For $w \geq 1$, let $r-1=r_{w-1} p^{w-1}+r_{w-2} p^{w-2}+$ $\ldots+r_{1} p+r_{0}$ with $r_{i} \in\{0,1, \ldots, p-1\}$ be the representation of $r-1$ in base $p$. Then we have: $r_{w-1}$ is the largest integer $i \geq 0$ for which $f^{i p^{w-1}} s \neq \mathbf{0}$.

Moreover, putting $t=f^{r_{w-1} p^{w-1}} s$ we have $t \in \mathcal{M}\left(f^{p^{w-1}}\right)$, $t$ has minimal polynomial $f^{r-r_{w-1} p^{w-1}}$ and period $N / p$.

Proof It is easy to see that $r_{w-1} p^{w-1} \leq r-1<\left(r_{w-1}+1\right) p^{w-1}$, hence $r_{w-1} p^{w-1}<$ $r \leq\left(r_{w-1}+1\right) p^{w-1}$. So $f^{r_{w-1} p^{w-1}}$ is not a characteristic polynomial of $s$, whereas $f^{\left(r_{w-1}+1\right) p^{w-1}}$ is. By Lemma 1(i), this means $f^{r_{w-1} p^{w-1}} s \neq \mathbf{0}$ and $f^{\left(r_{w-1}+1\right) p^{w-1}} s=$ $\mathbf{0 .}$ 
The last equality also means that $f^{p^{w-1}} t=\mathbf{0}$, i.e. $t \in \mathcal{M}\left(f^{p^{w-1}}\right)$. By Theorem 1 , $p^{w-1} \operatorname{ord}(f)$ is a period of $t$. By Lemma 3, we know ord $(f) \mid N^{\prime}$. Hence $p^{w-1} N^{\prime}=$ $N / p$ is also a period of $t$.

From Lemmas 3 and 4 we have:

Corollary 1 With the notations of Lemma $4: r_{w-1}=0$ iff $f^{p^{w-1}} s=\mathbf{0}$ iff $s \in$ $\mathcal{M}\left(f^{p^{w-1}}\right)$ iff $s$ has period $N / p$.

Based on the Lemmas and Corollary above we present the following two algorithms LinCompChar2 and LinComp given as Algorithms 1 and 2. The first is for $p=2$ and the second for arbitrary $p$ (including $p=2$ ). We formulated the algorithm for $p=2$ separately due to the importance of characteristic 2 and similarity with Games-Chan algorithm, as well as the fact that the code is somewhat simplified by the existence of only 2 cases at each step, corresponding to a $0 / 1$ digit in the binary representation of $r-1$, so the test in Corollary 1 is sufficient for determining the digit.

Note that throughout the algorithms the current value of the infinite sequence $s$ is implicitly stored as being the finite sequence $s^{\prime}=\left(s_{0}, s_{2}, \ldots, s_{N-1}\right)$ of length $N$, repeated periodically. When computing the action of a polynomial say $g=$ $\sum_{i=0}^{n} a_{i} x^{i}$ on the infinite $s$ thus stored, the result will be an infinite sequence $t$ of period $N$ stored as the finite sequence $t^{\prime}=\left(t_{0}, t_{2}, \ldots, t_{N-1}\right)$ consisting of the first $N$ terms of $t$, computed from $s^{\prime}$ as $t_{i}=\sum_{j=0}^{n} a_{j} s_{(i+j) \bmod N}$.

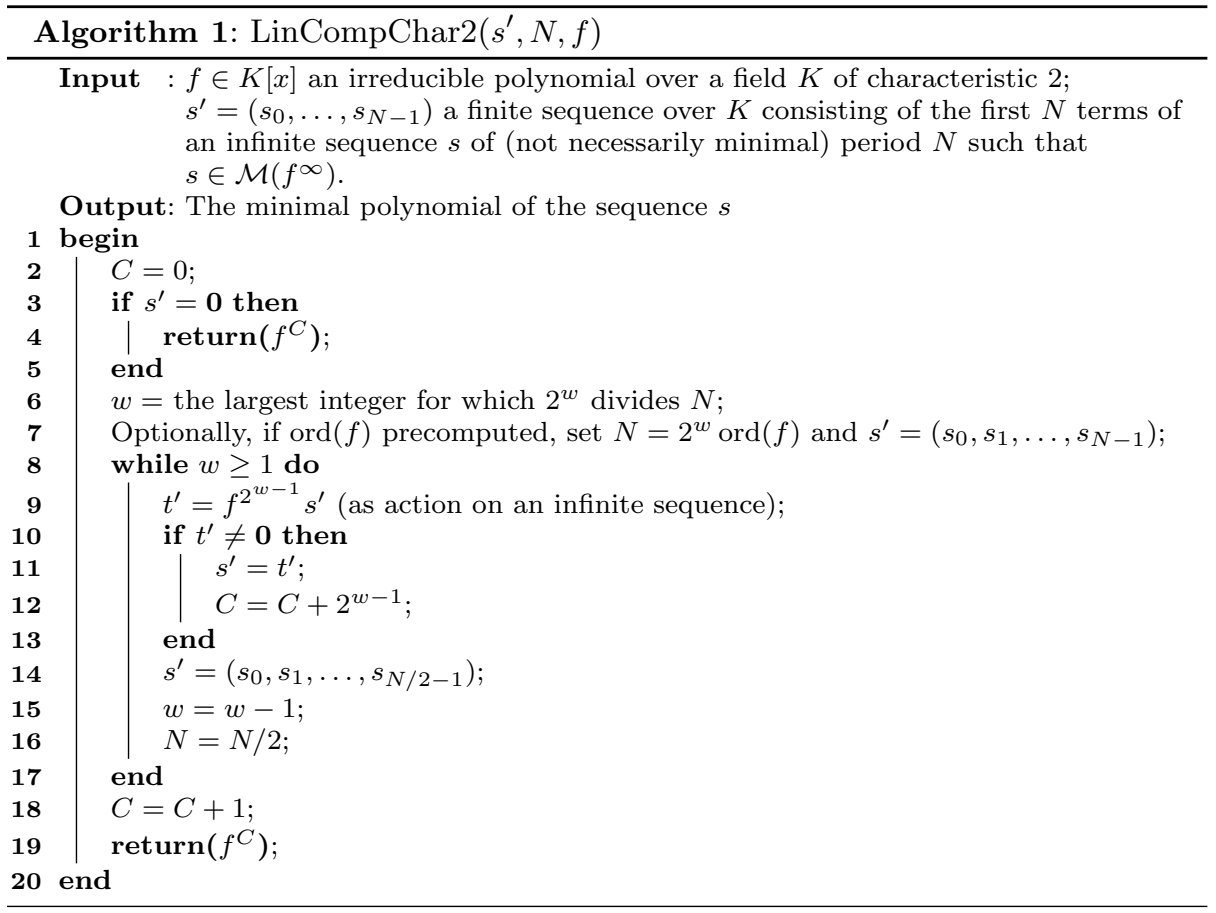




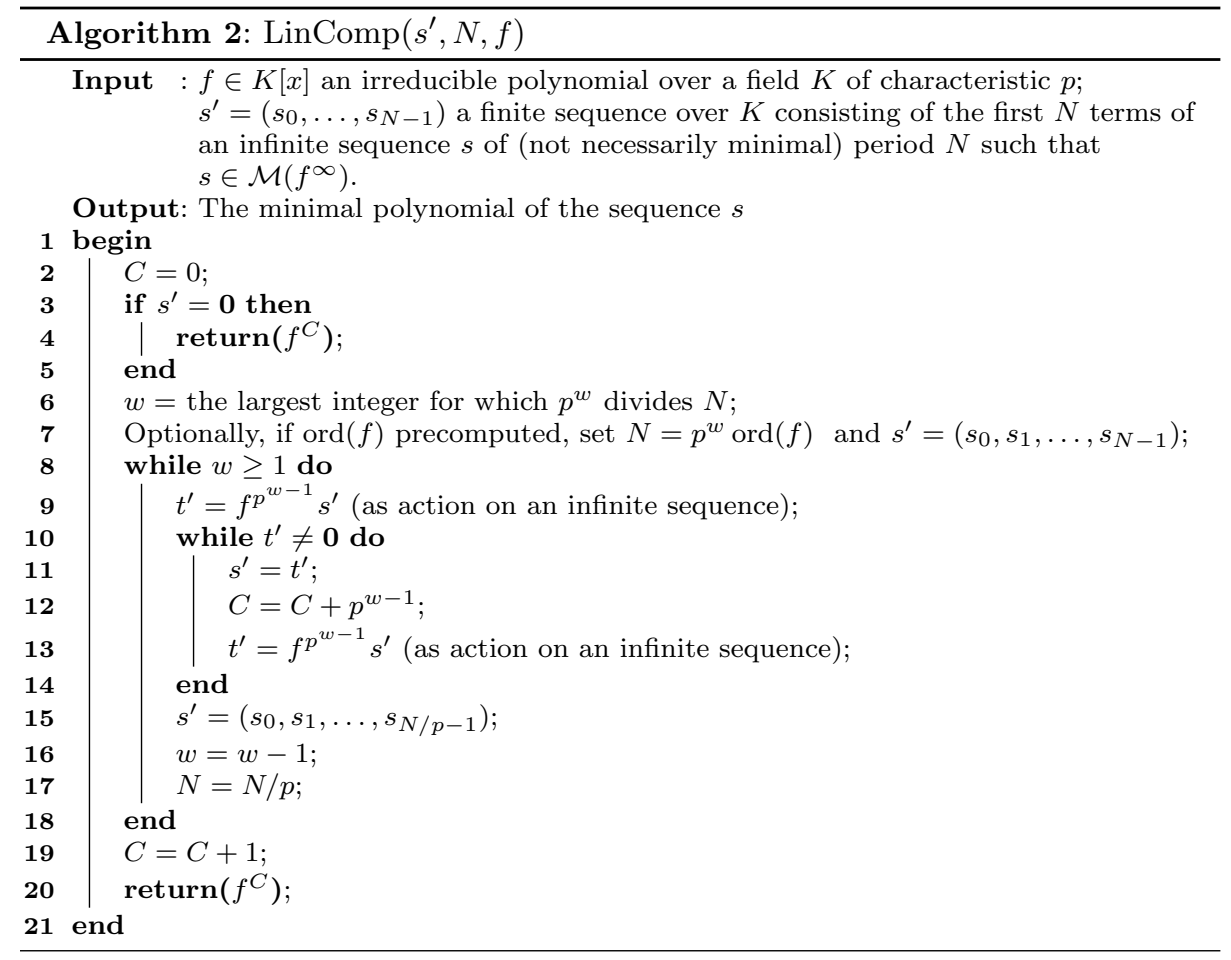

Theorem 2 Algorithms LinCompChar2 and LinComp (Algorithms 1 and 2) are correct and terminate.

Proof Let $r$ be such that $f^{r}$ is the minimal polynomial of the input sequence, i.e. the expected output of the algorithm. For the correctness of both algorithms we can prove the following invariants. At the start and at the end of each run of the outer while loop, $s^{\prime}$ is a finite sequence of length $N$, and $s^{\prime} \neq \mathbf{0}$. If we denote by $s$ the infinite sequence of period $N$ obtained by repeating $s^{\prime}$, we have $s \in \mathcal{M}\left(f^{p^{w}}\right)$ and the minimal polynomial of $s$ is $f^{r-C}$.

We outline the proofs for these invariants. The fact that $s^{\prime}$ is a finite sequence of length $N$ is immediate. The fact that $s^{\prime} \neq \mathbf{0}$ is obviously true before the while loop, due to the first if statement, line 3 . If $s^{\prime}$ is non-zero at the start of the outer while loop it will stay non-zero throughout, as each new value of $s^{\prime}$ is always set to either the first $N / p$ elements of $s^{\prime}$ (and by Corollary $1, s^{\prime}$ consists in this case of $p$ repeating identical sequences, which are therefore non-zero) or to a non-zero value of $t^{\prime}$.

The fact that $s \in \mathcal{M}\left(f^{p^{w}}\right)$ and the minimal polynomial of $s$ is $f^{r-C}$ holds before the outer while loop due to Lemma 3 and is then maintained due to to Lemma 4.

Finally, upon exiting the outer while loop, we have that $w=0$ so $s \in \mathcal{M}(f)$. Since $s^{\prime} \neq \mathbf{0}$, this means $f$ is the minimal polynomial of $s$. On the other hand we saw that the minimal polynomial of $s$ is $f^{r-C}$, therefore $1=r-C$ at this point, so $C+1$ is correctly returned as $r$. 
The termination follows from the fact that the value of $w$ is decreased by one at each run of the while loop. Additionally, for characteristic $p$, the inner while loop will run at most $p-1$ times, as we know that at the beginning of each run of the outer while loop we have $s \in \mathcal{M}\left(f^{p^{w}}\right)$ hence $f^{p^{w}} s=\mathbf{0}$.

Theorem 3 The complexity of Algorithms LinCompChar2 and LinComp (Algorithms 1 and 2) is $\mathcal{O}(N)$ if we consider $f$ to be fixed, or $\mathcal{O}(\operatorname{wt}(f) N)$ if $f$ is an input parameter.

Proof It suffices to show the second result. Let $N_{0}$ be the initial value of $N, w_{0}$ be the initial value computed for $w$ and let $N^{\prime}=N_{0} / p^{w_{0}}$. The (outer) while loop will run $w_{0}$ times, as shown in the proof of Theorem 2 .

In the binary case, the complexity of each individual loop is dominated by the calculation of $t^{\prime}=f^{2^{w-1}} s^{\prime}$, a finite sequence representing the first $2^{w} N^{\prime}$ terms of an infinite sequence of period $2^{w} N^{\prime}$. The number of summands for each term is fixed by Lemma 2 as $\operatorname{wt}(f)$. So the number of arithmetic operations is $2^{w} N^{\prime}(\operatorname{wt}(f)-$ 1). For characteristic $p$ each run of the outer while loop consists of at most $p$ computations of $t^{\prime}=f^{p^{w-1}} s^{\prime}$, each taking $(\operatorname{wt}(f)-1) p^{w} N^{\prime}$ steps.

In total we have $\sum_{w=1}^{w_{0}} 2^{w} N^{\prime}(\operatorname{wt}(f)-1)=2\left(2^{w_{0}}-1\right) N^{\prime}(\operatorname{wt}(f)-1)<2 N_{0} \operatorname{wt}(f)$ for the binary case and $(\operatorname{wt}(f)-1) N^{\prime} \sum_{w=1}^{w_{0}} p^{w+1}=(\operatorname{wt}(f)-1) p^{2} N^{\prime} \frac{p^{w_{0}}-1}{p-1}<$ $\frac{p^{2}}{p-1} \operatorname{wt}(f) N_{0}$ for arbitrary $p$.

Alternative algorithms can be obtained for LinCompChar2 and LinComp (Algorithms 1 and 2) by using the last equivalence of Corollary 1. Namely, we can check immediately at the start of the outer while loop whether the current value of $s^{\prime}$ consists of $p$ repeating copies of the same sequence. If this is the case we do not compute $t^{\prime}$ but skip to the instructions for updating the values of $s^{\prime}, w$ and $N$ at the end of the loop. The algorithms thus modified would have the same worst-case complexity but will behave slightly better for the case when $r-1$ has many 0's in its representation in base $p$. Since each digit has a $1 / p$ chance of being 0 , the savings will be more significant in characteristic 2 . We present an alternative to Algorithm 1 as Algorithm 3.

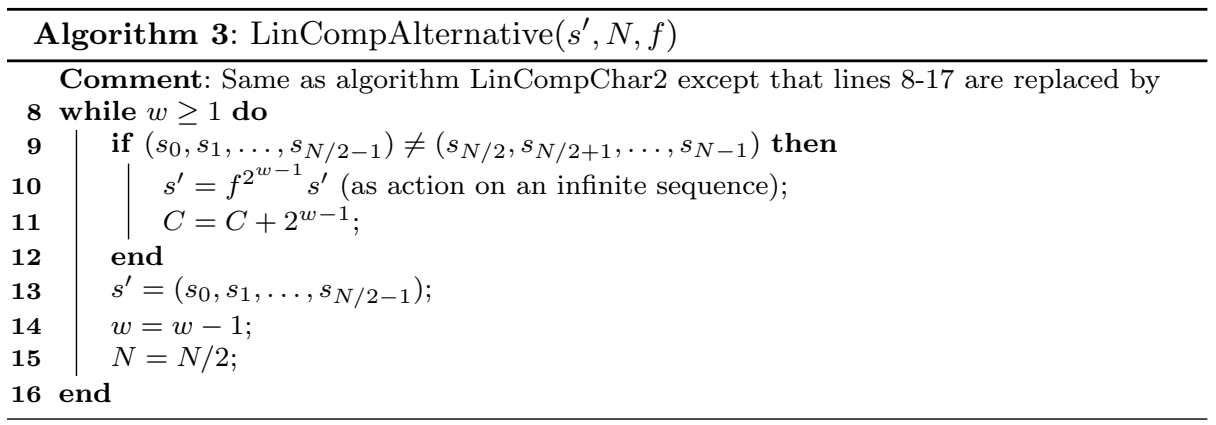

Remark 1 For $f=x-1$, Algorithm 1 reduces to the Games-Chan algorithm, [3]. Firstly, ord $(f)=1$, so we can put $N=2^{w}$ in line 7 . Secondly, computing $t^{\prime}=(x-1)^{2^{w-1}} s^{\prime}=\left(x^{2^{w-1}}-1\right) s^{\prime}$ for a sequence of period $2^{w}$ means $t^{\prime}$ is the 
component-wise subtraction of the two halves of $s^{\prime}$ (i.e. $t^{\prime}$ is $L(s)-R(s)$ if $L(s)$ and $R(s)$ denote the left and right half of $s$, as in the notation used in the Games-Chan algorithm). Therefore checking whether $t^{\prime}=\mathbf{0}$ will in this case mean checking whether the two halves of $s^{\prime}$ are identical. Algorithm 3 will also become virtually the same as Algorithm 1 and the same as Games-Chan algorithm in this situation. Similarly, for $f=x-1$ Algorithm 2 reduces to the algorithm of Ding et al. [2].

Note that in the Games-Chan algorithm the final instruction $C=C+1$ is done conditionally, only if $s^{\prime} \neq \mathbf{0}$. If one deals at the start of the algorithm with the case of an all-zero input sequence (as we do), it is no longer necessary to check at the end if $s^{\prime} \neq \mathbf{0}$, as this will always be the case (see the proof of Theorem 2).

Example 1 Let $K=\mathrm{GF}(2)$ and $f=x^{3}+x+1$. The sequence $s \in \mathcal{M}\left(f^{\infty}\right)$ has period $N=28$ and its first 28 terms are $s^{\prime}=0000000010110000101110111011$. The running of Algorithm 1 is described in Table 1.

Table 1 Example run for Algorithm 1

\begin{tabular}{|c|c|c|c|}
\hline$s^{\prime}$ & $\mathrm{w}$ & $t=\mathbf{0} ?$ & $\mathrm{C}$ \\
\hline 00000000101100 & 2 & No & 2 \\
00101110111011 & & & \\
\hline 00101110010111 & 1 & Yes & 2 \\
\hline 0010111 & 0 & & \\
\hline \multicolumn{3}{|c|}{ return $\left(f^{3}\right)$} & 3 \\
\hline
\end{tabular}

\section{Linear Complexity of Finite Sequences which have a Characteristic Polynomial equal to a Power of an Irreducible Polynomial}

It was noticed by Sălăgean in [7] and by Meidl in [6] that we actually do not need to have the whole period of the infinite sequence in the Games-Chan algorithm in order to compute the linear complexity. In this section we generalise the idea of Meidl, [6, Sections 2 and 3], by using the initial terms of a sequence, and knowledge of a characteristic polynomial to treat it as an infinite sequence.

For a fixed polynomial $g$, an individual infinite sequence $s$ with characteristic polynomial $g$ is uniquely defined (within the class of all sequences with characteristic polynomial $g$ ) by its initial $\operatorname{deg}(g)$ terms. Can we decide if $s$ admits a characteristic polynomial of lower degree just by examining these initial $\operatorname{deg}(g)$ terms?

Lemma 5 Let $s$ be an infinite sequence with characteristic polynomial $g=g_{1} g_{2}$ with $g_{1}, g_{2}$ monic. Then:

$s$ has characteristic polynomial $g_{1}$ iff the finite sequence $s^{\prime}=\left(s_{0}, \ldots, s_{\operatorname{deg}(g)-1}\right)$ has characteristic polynomial $g_{1}$.

Proof The direct implication is obvious. Conversely, assume $s^{\prime}$ has characteristic polynomial $g_{1}$ i.e. $g_{1} s^{\prime}=(0, \ldots, 0)$, a finite sequence of $\operatorname{deg}(g)-\operatorname{deg}\left(g_{1}\right)=\operatorname{deg}\left(g_{2}\right)$ terms. Note this sequence also coincides with the first $\operatorname{deg}\left(g_{2}\right)$ terms of the infinite sequence $g_{1} s$. By Lemma $1(\mathrm{i}), g s=g_{2} g_{1} s=\mathbf{0}$, so $g_{1} s$ has characteristic polynomial 
$g_{2}$. But then $g_{1} s=\mathbf{0}$ as its first $\operatorname{deg}\left(g_{2}\right)$ terms are all zero and its linear complexity is at most $\operatorname{deg}\left(g_{2}\right)$.

Consequently, if we are given $s^{\prime}$ as being the first $v \operatorname{deg}(f)$ terms of a sequence $s \in \mathcal{M}\left(f^{v}\right)$ we can check whether $s$ admits some characteristic polynomial of lower degree, i.e. $f^{v^{\prime}}$ with $v^{\prime}<v$ by checking whether $f^{v^{\prime}} s^{\prime}=\mathbf{0}$. Again, a $p$-ary search will make it more efficient.

The algorithm LinCompFinite is given as Algorithm 4 and is similar to the Algorithm 2 in the previous section. Note that throughout the algorithm, the length of the current value of $s^{\prime}$ is $v \operatorname{deg}(f)$ for the current value of $v$.

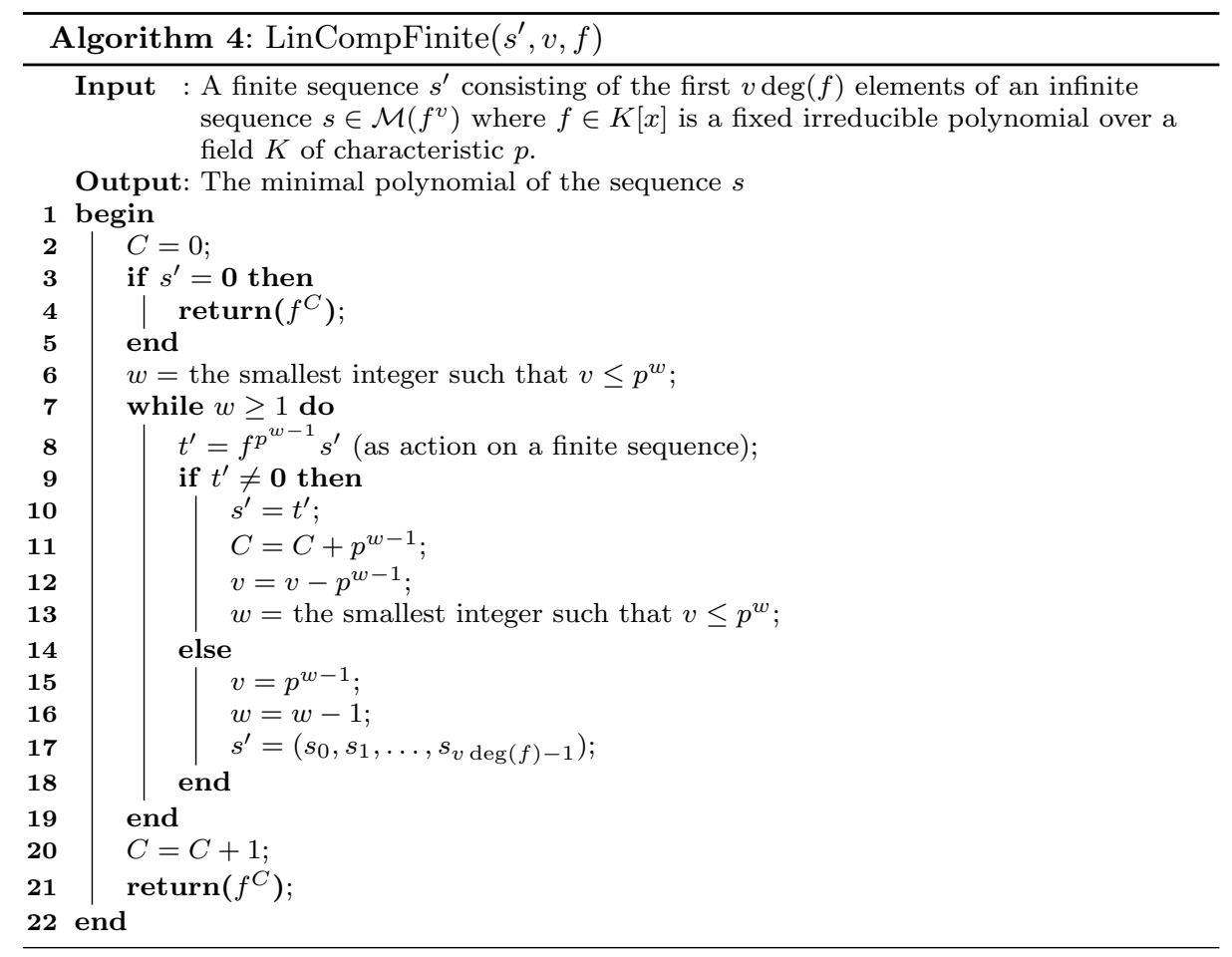

Theorem 4 Algorithm 4 is correct and terminates.

Proof Let $s^{(0)}$ be the original value of the infinite input sequence $s$ and let $r$ be such that $f^{r}$ is its minimal polynomial. For the correctness, we will show that throughout the algorithm $s^{\prime}$ consists of the first $v \operatorname{deg}(f)$ terms of $f^{C} s^{(0)}$ and $p^{w} \geq v \geq r-C \geq 1$, with $w$ minimal such that $p^{w} \geq v$. Therefore $s^{\prime}$ in conjunction with the characteristic polynomial $f^{v}$ correctly defines the infinite sequence $f^{C} s^{(0)}$. All these statements are obviously true before the while loop begins. We will assume they are true at the start of a run of the loop and show they are true at the end of the loop.

If the $t^{\prime} \neq \mathbf{0}$ branch of the if is taken, then $v_{\text {new }}=v_{\text {old }}-p^{w_{\text {old }}-1} \geq r-$ $C_{\text {old }}-p^{w_{\text {old }}-1}=r-C_{n e w}$, where the old and new indices refer to the value at the 
Table 2 Example run for Algorithm 4

\begin{tabular}{|c|c|c|c|c|}
\hline$s^{\prime}$ & $\mathrm{w}$ & $\mathrm{v}$ & $t=\mathbf{0} ?$ & $\mathrm{C}$ \\
\hline 010100001011010110 & 3 & 6 & Yes & 0 \\
\hline 010100001011 & 2 & 4 & No & 2 \\
\hline 001111 & 1 & 2 & No & 3 \\
\hline 101 & 0 & 1 & & \\
\hline \multicolumn{5}{|c|}{ return $\left(f^{4}\right)$} \\
\hline
\end{tabular}

beginning and at the end of the loop. In addition, $r-C_{n e w} \geq 1$ : if we assume the contrary, i.e. $r \leq C_{n e w}$, then $f^{C_{n e w}}$ would be a characteristic polynomial for $s^{(0)}$, so $f^{C_{n e w}} s^{(0)}=\mathbf{0}$. But then $s_{n e w}^{\prime}$, consisting of the first terms of $f^{C_{n e w}} s^{(0)}$, would equal $\mathbf{0}$. On the other hand $s_{n e w}^{\prime}=t^{\prime} \neq \mathbf{0}$, contradiction.

If the else branch of the if is taken, we know $f^{p^{w_{o l d}-1}} s_{\text {old }}^{\prime}=\mathbf{0}$. By Lemma 5 , this means $f^{C_{\text {old }}} s^{(0)}$ has characteristic polynomial $f^{p^{w_{\text {old }}-1}}=f^{v_{\text {new }}}$, so $v_{\text {new }} \operatorname{deg}(f)$ of its initial terms are sufficient to determine the sequence. Moreover, since $f^{r-C_{\text {old }}}$ is the minimal polynomial of $f_{\text {old }}^{C} s^{(0)}$ we have $p^{w_{\text {old }}-1} \geq r-C_{\text {old }}$. Hence $v_{\text {new }}=$ $p^{w_{\text {old }}-1} \geq r-C_{\text {old }}=r-C_{\text {new }}$.

Finally, upon exiting the while loop we have $w=0$ and since $1=p^{w} \geq v \geq$ $r-C \geq 1$, it follows that $r-C=1$, so $C+1$ is correctly returned as $r$.

To show termination, note that $v$ decreases throughout the algorithm.

Theorem 5 Algorithm 4 run on a sequence of length $m$ has complexity $\mathcal{O}(m)$ for a fixed $f$, or $\mathcal{O}(m \operatorname{wt}(f))$ if $f$ is an input parameter.

Proof We can see that each run of the while loop takes $v \operatorname{deg}(f)(\operatorname{wt}(f)-1)$ steps for the current value of $v$, which is upper bounded by $p^{w}$. In each run of the loop, the value of $w$ is decreased or stays the same, but can only stay the same for at most $p$ successive runs of the while loop. So if $w_{0}$ is the initial value of $w$, we have at most $\sum_{w=1}^{w_{0}} p^{w+1} \operatorname{deg}(f)(\operatorname{wt}(f)-1)=\frac{p^{2}}{p-1}\left(p^{w_{0}}-1\right) \operatorname{deg}(f)(\operatorname{wt}(f)-1) \leq$ $\frac{p^{3}}{p-1} v \operatorname{deg}(f) \operatorname{wt}(f)$ steps.

Example 2 Let $K=G F(2)$ and $f=x^{3}+x+1$. The finite sequence $s^{\prime}=010100$ 001011010110 consists of the first $6 \operatorname{deg}(f)=18$ terms of a sequence $s \in \mathcal{M}\left(f^{6}\right)$. The running of Algorithm 4 is described in the Table 2.

Remark 2 Berlekamp-Massey algorithm has quadratic complexity. When we consider $f$ fixed, Algorithm 4 has linear complexity, so it is clearly more efficient. Let us compare the two algorithms when $f$ is an input parameter. Assume the minimal polynomial of $s$ will turn out to be $f^{r}$. Assume both algorithms are run on a finite sequence $s$ of length $m$, so we need that the length $m$ satisfies $m \geq v \operatorname{deg}(f)$ for Algorithm 4 (where $v$ is some upper bound on $r$ known apriori) and $m \geq 2 r \operatorname{deg}(f)$ for the Berlekamp-Massey algorithm. The computational complexity will be $\mathcal{O}(m(\operatorname{wt}(f)-1))$ for Algorithm 4 (see proof of Theorem 5 ) and $\mathcal{O}\left(m \operatorname{deg}\left(f^{r}\right)\right)$ for the Berlekamp-Massey algorithm. (A finer bound for the latter would be $\mathcal{O}(m(z-1))$ where $z$ is an upper bound on the weight of the minimal polynomial as well as the weights of all the intermediate minimal polynomials of the sequence. We have $z-1 \leq \operatorname{deg}\left(f^{r}\right)$, and in the worst case equality is attained, for example in characteristic 2 , for $f=x+1$ and $r=2^{n}-1$ one can check that 
$\operatorname{wt}\left((x+1)^{2^{n}-1}\right)-1=\operatorname{deg}\left((x+1)^{2^{n}-1}\right)$.) Since $\operatorname{wt}(f)-1 \leq \operatorname{deg}(f) \leq \operatorname{deg}\left(f^{r}\right)$, Algorithm 4 is in general more efficient than Berlekamp-Massey. However, we must note that this comes at the cost of requiring the a priori knowledge of a characteristic polynomial of $s$.

Remark 3 For $f=x-1, p=2$ and arbitrary $v$, our Algorithm 4 reduces to Algorithm 1 of [6]. For $f=x^{2}+x+1$ and $v$ being a power of 2 , it reduces to Algorithm 2 in [6] (which, as remarked at the end on Section 3 of [6] could be generalised to arbitrary $f$ and $v$ a power of 2 ).

Let us examine the relation between the algorithms in this section (where a finite portion of the sequence is known) and the ones in the previous section (where a whole period of the sequence is known, i.e. the whole infinite sequence is known). We could easily transform one problem into the other, namely, if we have a finite sequence we can generate the whole period using the given characteristic polynomial $f^{v}$ (note that this process can take a number of steps exponential in the length of the original input finite sequence, see discussion further on). Conversely given an infinite sequence of period $N$ we could restrict to the initial $p^{w} \operatorname{deg}(f)$ terms (with $w$ maximal such that $p^{w} \mid N$ ), as $f^{p^{w}}$ is guaranteed by Lemma 3 to be a characteristic polynomial of $f$. We compare now the complexities of the two types of algorithm. Algorithms 1, 2 and 3 of the previous section are $\mathcal{O}(N \mathrm{wt}(f))$, and if $\operatorname{ord}(f)$ is known, it is $\mathcal{O}\left(p^{w} \operatorname{ord}(f) \operatorname{wt}(f)\right)$ (as $N$ can be replaced by $p^{w} \operatorname{ord}(f)$, see line 7 in the algorithms). On the other hand, Algorithm 4 of this section has complexity $\mathcal{O}(v \operatorname{deg}(f)$ wt $(f))$, so if we know no better bound than $v=p^{w}$ for the exponent of $f$ (as is the case in the previous section), this becomes $\mathcal{O}\left(p^{w} \operatorname{deg}(f) \operatorname{wt}(f)\right)$. Since $\operatorname{deg}(f) \leq \operatorname{ord}(f) \leq p^{\operatorname{deg}(f)}-1$ with both lower and upper bounds attained for particular values of $f$, it means that the algorithms of the previous section are no better, and potentially exponentially slower than the ones in this section (to clarify, all are linear in the size of the input, but the size of the input can be exponentially higher if we use the full period rather than the initial $v \operatorname{deg}(f)$ terms). So the algorithms of the previous section should be avoided in favour of the one in this section. We did nevertheless present the former as they are direct generalisations of the Games-Chan algorithm.

Remark 4 Since the algorithm developed here is a generalization of the ones given by Meidl in [6], it may seem natural to go further and adapt these algorithms into ones capable of determining the $k$-error linear complexity, as a generalization of the work carried out in [6], Section 4. However, we do not believe that such work would be worthwhile for the following reason:

In [7] the following definition is given for the $k$-error complexity of a finite sequence, $z$, of length $t$ with respect to a set $A$ of infinite sequences:

$$
c_{k}(z, A)=\min \left\{c(s) \mid s \in A, \operatorname{wt}\left(\left(s_{0}, s_{1}, \ldots, s_{n-1}\right)-z\right) \leq k\right\}
$$

where $c(t)$ is the complexity of the infinite sequence $s$. This definition is used in [7] with the set $A$ being the set of all sequences with period a power of 2 . This is because, if it is known that the initial finite sequence was part of an infinite sequence $s$ whose period $N$ was a power of 2, introducing errors to this infinite sequence will not affect this property (because the error sequence will have the same period as $s$, i.e. a power of two and hence adding them to the sequence will again result in a sequence with period a power of two), and so $c_{k}(z, A)=c_{k}\left(z, \mathcal{S}_{N}\right)$ 
where $\mathcal{S}_{N}$ is the set of all binary sequences o period $N$. However, this is not always the case, and specifically, it is not the case in the way the definition is used in [6] Section 4.

In [6] Section 4, the same general definition is used, although now the set $A$ is defined to be $\mathcal{M}\left(\left(x^{2}+x+1\right)^{2^{v}}\right)$. However, $s \in \mathcal{M}\left(\left(x^{2}+x+1\right)^{2^{v}}\right)$ does not imply $(s+e) \in \mathcal{M}\left(\left(x^{2}+x+1\right)^{2^{v}}\right)$, for all sequences $e$ of the same period $N$ as $s$. Hence in this case $c_{k}\left(s, \mathcal{M}\left(\left(x^{2}+x+1\right)^{2^{v}}\right)\right) \neq c_{k}\left(s, \mathcal{S}_{N}\right)$. Therefore while the algorithm presented in [6] Section 4 does calculate $c_{k}\left(s, \mathcal{M}\left(\left(x^{2}+x+1\right)^{2^{v}}\right)\right)$, this is not equal to the $k$-error linear complexity (in the classical sense) of the input sequence $s$. Therefore we do not feel it is worthwhile adapting the algorithm presented in this paper to the $k$-error linear complexity problem, as it would suffer from the same restriction.

\section{Linear Complexity of Infinite Sequences which have a Characteristic Polynomial equal to a Product of Known Irreducible Factors}

With a simple adjustment to the algorithms in Section 4, we can greatly increase their scope, so that they can be applied to any sequence provided each of the irreducible factors of a characteristic polynomial are known.

As a consequence of Lemma 1(ii) we have:

Corollary 2 Assume that the minimal polynomial of a sequence $s$ is of the form $f_{1}^{r_{1}} f_{2}^{r_{2}} \ldots f_{k}^{r_{k}}$, with $f_{i}$ distinct irreducible polynomials. Let $v_{i} \geq r_{i}$ for $i=2, \ldots, k$. Then $f_{1}^{r_{1}}$ is a minimal polynomial of the sequence $f_{2}^{v_{2}} \ldots f_{k}^{v_{k}} s$.

Therefore, if we know each of the irreducible factors of a characteristic polynomial of a sequence and we have an upper bound on the powers of each irreducible polynomial, we can use Corollary 2 and Algorithm 4 to successively determine the powers of each irreducible polynomial in the minimal polynomial. The resulting algorithm LinCompSet is presented as Algorithm 5.

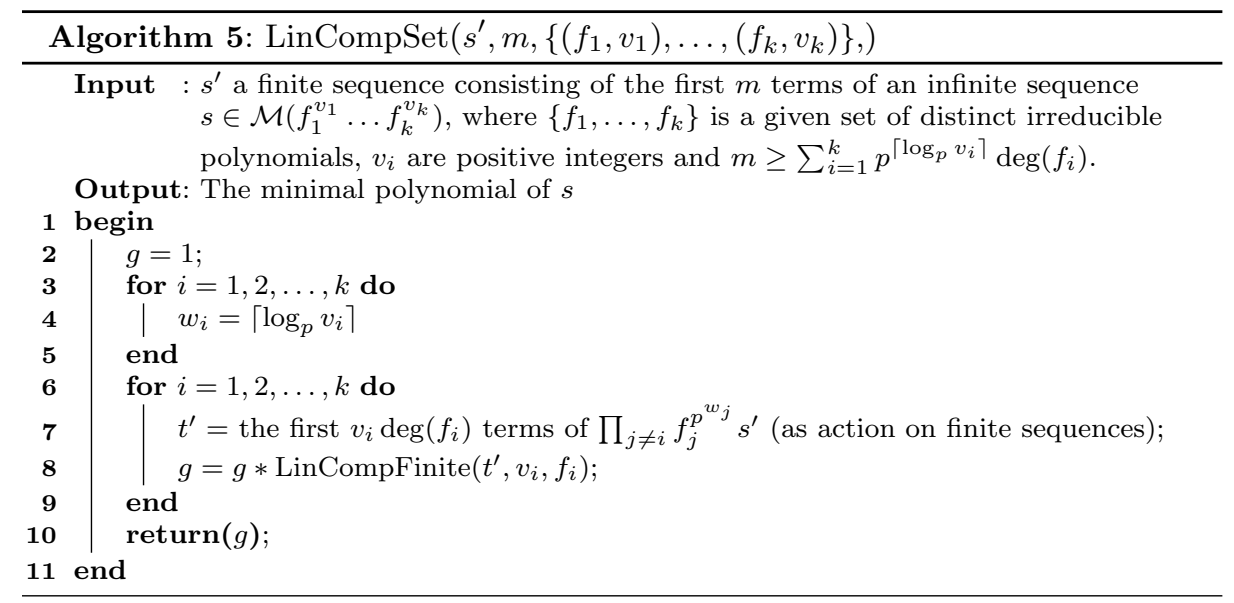


Theorem 6 For a sequence of length $m$, and a fixed set $\left\{f_{1}, \ldots, f_{k}\right\}$, Algorithm 5 has complexity $\mathcal{O}(m)$. For a general set of $k$ elements $\left\{f_{1}, \ldots, f_{k}\right\}$, the algorithm will have complexity $\mathcal{O}\left(\left(k m \sum_{i=1}^{k} \operatorname{wt}\left(f_{i}\right)\right)\right)$.

Proof In each of the $k$ runs of the outer for loop, the computation of $t^{\prime}$ takes $m\left(\left(\sum_{j=1}^{k}\left(\operatorname{wt}\left(f_{j}\right)-1\right)\right)-\left(\operatorname{wt}\left(f_{i}\right)-1\right)\right)$ operations if we do a straightforward implementation. One can obtain a more efficient implementation by reusing intermediate results from the computation of $t^{\prime}$ for different values of $i$, but none of the versions we considered achieved an improved $\mathcal{O}$ complexity class. LinCompFinite has complexity $\mathcal{O}\left(v_{i} \operatorname{wt}\left(f_{i}\right) \operatorname{deg}\left(f_{i}\right)\right)$, more precisely at most $\frac{p^{2}}{p-1}\left(p^{w_{i}}-1\right) \operatorname{deg}\left(f_{i}\right)\left(\operatorname{wt}\left(f_{i}\right)-1\right)$ steps (see the proof of Theorem 3). Since $m \geq p^{w_{i}} \operatorname{deg}\left(f_{i}\right)$, this gives a total of at most $m \frac{p^{2}}{p-1} \sum_{j=1}^{k}\left(\operatorname{wt}\left(f_{j}\right)-1\right)$ i.e. $\mathcal{O}\left(m \sum_{i=1}^{k} \operatorname{wt}\left(f_{i}\right)\right)$ for each of the $k$ loops.

Note that Algorithm 5 is therefore efficient only if $\left\{f_{1}, \ldots, f_{k}\right\}$ has a small cardinality $k$ and the total weight of its elements is small.

In the algorithm we assumed that the irreducible polynomials $f_{1}, \ldots, f_{k}$ as well as the bounds $v_{1}, \ldots, v_{k}$ are known apriori. If this is not the case, but we do know that $s$ has period $N$, we can write $N=p^{w} N^{\prime}$ with $p \nmid N^{\prime}$ and we know that the minimal polynomial of $s$ is a factor of $\left(x^{N^{\prime}}-1\right)^{p^{w}}$, i.e. all the irreducible factors of the minimal polynomial are factors of $x^{N^{\prime}}-1$ and have multiplicity at most $p^{w}$. Therefore we can put $\left\{f_{1}, \ldots, f_{k}\right\}=\left\{f \in K[x] \mid f\right.$ irreducible factor of $\left.x^{N^{\prime}}-1\right\}$ and $v_{i}=p^{w}$ for all $i$. Consequently we would need $m=N$. In this case, the algorithm is efficient when $N^{\prime}$ is a relatively small constant, or when $x^{N^{\prime}}-1$ has only a few factors, and their weight is small. Here is such an example:

Example 3 Consider a binary sequence $s^{\prime}$ of length $m$ and $\left\{f_{1}, f_{2}, f_{3}\right\}=\left\{x+1, x^{3}+\right.$ $\left.x^{2}+1, x^{3}+x+1\right\}$. Assume we are also given values for $v_{i}$. (Alternatively, assume we know $s^{\prime}$ is part of a sequence of period $N=2^{w} * 7$, so we can put $v_{i}=2^{w}$ and $\left\{f_{1}, f_{2}, f_{3}\right\}=\left\{x+1, x^{3}+x^{2}+1, x^{3}+x+1\right\}$, as $x^{7}-1=(x+1)\left(x^{3}+x^{2}+\right.$ 1) $\left(x^{3}+x+1\right)$.) The number of operations in Algorithm 5 will be about $16 \mathrm{~m}$ (see proof of Theorem 6), whereas Berlekamp-Massey's algorithm would need $\operatorname{deg}(\mathrm{g}) \mathrm{m}$ operations, where $g$ is the minimal polynomial of $s$. So in this example, and for large enough degree of $g$, Algorithm 5 is more efficient.

In the worst case Algorithm 5 becomes less efficient than Berlekamp-Massey's algorithm. The number of operations in Algorithm 5 is at least $k^{2} m$. We examine cases where $k$ is large. For the particular case when $N^{\prime}=2^{n}-1$ is a (Mersenne) prime and $m=N=p^{w} N^{\prime}$ with $w$ a small constant, we have $k=\frac{N^{\prime}-1}{n}+1$ (the factors of $x^{2^{n}-1}-1$ are in this case $x-1$ and the $\varphi\left(2^{n}-1\right) / n=\left(2^{n}-2\right) / n$ primitive polynomials of degree $n$ as in [5, Theorem 3.5]). Since in this case $k \approx \frac{N}{\log N}$, the complexity of the algorithm becomes in the worst case $\Omega\left(N^{3} / \log ^{2} N\right)$ i.e. more than quadratic.

\section{Conclusion}

We proposed algorithms for computing the linear complexity and minimal polynomial for sequences which admit as a characteristic polynomial a power of a fixed irreducible polynomial $f$. They work for any field of finite characteristic and we 
do not necessarily need the whole period of the sequence. For $f=x-1$ our algorithms reduce to the algorithms of Games-Chan [3], Ding et al. [2] and Meidl [6]. We can also apply our algorithms to the case where the characteristic polynomial is a product of powers of a small number of known irreducible polynomials. All our algorithms have linear computational complexity (when assuming the irreducible polynomials are fixed).

\section{References}

1. A. J. Burrage, A. Sălăgean and R. C.-W. Phan, "Linear Complexity for Sequences with Characteristic Polynomial $f^{v}$ ", Proceedings of IEEE International Symposium on Information Theory (ISIT), pp.688-692, St. Petersburg, Russia, 2011.

2. C. Ding, G. Xiao and W. Shan, The Stability Theory of Stream Ciphers. Springer Verlag, 1991.

3. R. Games and A. Chan, "A fast algorithm for determining the complexity of a binary sequence with period $2^{n}$, IEEE Trans. Information Theory, vol. 29, pp. 144-146, 1983.

4. S. W. Golomb. Shift Register Sequences. Aegean Park Press, 1982.

5. R. Lidl and H. Niederreiter, Introduction to Finite Fields and Their Applications. Cambridge university Press, 1994.

6. W. Meidl, "How to determine linear complexity and $k$-error linear complexity in some classes of linear recurring sequences", Cryptography and Communications, vol. 1, pp. 117133,2009

7. A. Sălăgean, "On the computation of the linear complexity and the k-error linear complexity of binary sequences with period a power of two", IEEE Trans. Information Theory, vol. 51, pp. 1145-1150, 2005. 